\title{
ALRE stakeholder survey analysis key findings
}

This summary shares the results of a stakeholder survey on the policy issues and demand for evidence related to inclusive agricultural commercialisation across Africa by the Accompanied Learning for Relevance and Effectiveness (ALRE) team of the Agricultural Policy Research in Africa (APRA) programme. The survey collected data between January and March 2020 on a series of closed questions to i) understand the top five policy priorities to support agricultural commercialisation and the most effective communication methods; and ii) the different factors and the role of research to influence inclusive agricultural commercialisation.

\section{Priority issues requiring better-quality evidence and analysis}

Of the 16 potential issues included in the survey, those identified as the highest priority requiring better-quality evidence and analysis were: improved markets for smallholder products; credit and financial services; the empowerment of youth and women in agriculture; and rural extension and advisory services.

From the respondents' perspective from the APRA focal regions:

East Africa-based respondents viewed agro-input policies as the highest priority issue, and viewed credit and financial services as a lower priority than other regions.

- Southern African respondents viewed land reform as a higher priority issue than other regions.

- The priority issues for West African respondents were broadly aligned to the priorities across all stakeholders.

\section{Methods required for communicating research}

The top four methods of communicating research that surveyed stakeholders require more of were: workshops and seminars with experts; face-to-face briefings; commissioned technical reviews; and succinct policy briefs.

This view was reflected across the APRA focal regions. Most of these methods involve stronger engagement with experts and policymakers and require time and financial resources. It is important to note that these results were generated pre-COVID-19 and further reflection is needed to understand the implications of the pandemic on methods to support research-policy engagement.

Methods which had less demand were: written media, including newspaper articles and academic research papers, and internet content, including blogs and social media, thematic emailed newsletters and focused websites.

Disaggregating respondents by their field of work revealed exceptions to the overall results. For instance, 63 per cent of policymakers selected succinct policy briefs in their top three methods, whilst 50 per cent of the technical staff responsible for implementing policy preferred more in-depth methods of communicating research, such as commissioned technical reviews.

\section{Has research on agricultural policy and markets contributed to increased inclusive agricultural commercialisation?}

Respondents reviewed the impact of research on the role of four key factors for increasing inclusive agricultural commercialisation in Africa: the private sector, policies and regulations, farmer organisations, and market dynamics. They perceived these factors to have marginally improved towards supporting inclusive commercialisation over the last three years.

Respondents in West Africa perceived research as having contributed most significantly to the improved role of each key factor in supporting inclusive agricultural commercialisation. Respondents from East Africa viewed research as slightly less influential, whereas respondents from Southern Africa perceived research as having significantly less influence on improvements than other regions.

The low perception of the contribution of research to support market dynamics' supportiveness reinforces the finding above that improved markets for smallholder products was by far the highest-ranking priority issue requiring better-quality evidence. This is particularly true for respondents in Southern Africa. 


\section{Role of evidence in policies to support inclusive agricultural commercialisation}

\section{Proportion of respondents from each APRA focal region}

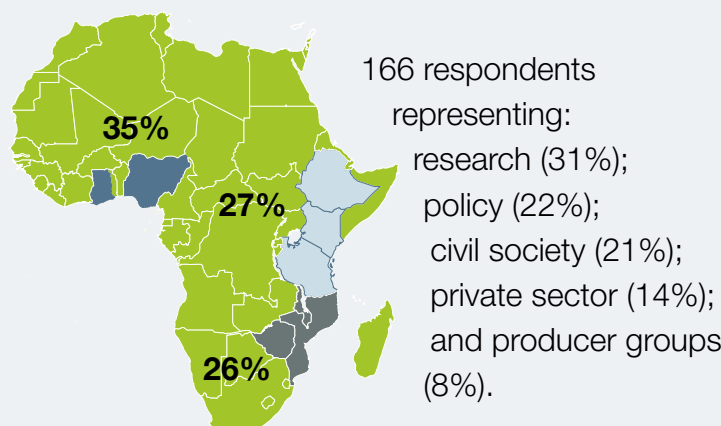

\section{Communicating research}
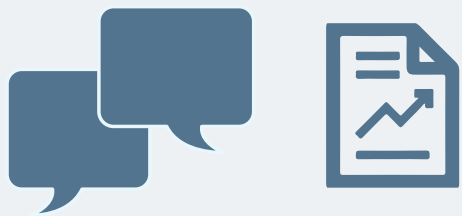

Workshops, expert seminars and briefings ranked as the most effective communication methods. Technical reviews and succinct policy briefs followed.

Online content (blogs, newsletters, websites) ranked least effective.

\section{Top 5 policy issues requiring better quality evidence}

1. Improved markets for smallholder products

2. Credit and financial services

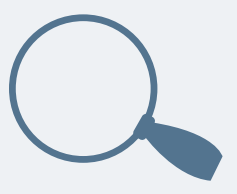

3. Empowerment of youth and women in agriculture

4. Rural extension and advisory services

5. Agro-input policies

\section{Top 4 factors supporting inclusive agricultural commercialisation}

1. Farmers organisations

2. Private sector

3. Policies and regulations

4. Market dynamics

The private sector was the most improved in the past three years.

Survey results suggest only a marginal contribution of evidence across all factors

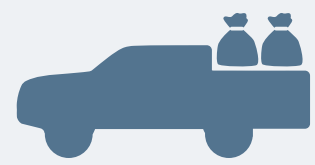

\section{Follow up questions for focus group discussions}

- How has COVID-19 affected these insights and the evidence needs of policymakers?

- What are the new opportunities and challenges created by COVID-19 to make agricultural commercialisation more inclusive?

What evidence is needed to continue to promote the inclusion of women and girls in: farmer organisations; private sector initiatives; policies and regulations and market dynamics?

- How can we most effectively communicate evidence in the context of COVID-19?

This is an Open Access report distributed under the terms of the Creative Commons Attribution Non Commercial No Derivatives 4.0 International licence (CC BY-NC-ND), which permits use and distribution in any medium, provided the original authors and source are credited, the work is not used for commercial purposes, and no modifications or adaptations are made. 